\title{
Atmospheric effects on availability of free space optics systems
}

\author{
Ales Prokes \\ Brno University of Technology \\ Department of Radio Electronics \\ Purkynova 118 \\ 61200 Brno \\ Czech Republic \\ E-mail: prokes@feec.vutbr.cz
}

\begin{abstract}
The availability of free space optics (FSO) systems in dependence on weather conditions and on FSO link parameters, such as transmitted optical power, beam divergence, receiver sensitivity or link path distance, is discussed. A number of phenomena in the atmosphere, such as absorption, scattering, and turbulence, can affect beam attenuation, but in the case of wavelengths typical of FSO systems operation, only scattering and turbulence are appropriate to be taken into consideration. We model the power loss caused by turbulence by using the Rytov scintillation theory. Attenuation due to scattering, which can be expressed as a function of the link distance, wavelength, and meteorological visibility, is calculated from visibility data collected at several airports in Europe. Statistical evaluation of the attenuation caused by scattering and the power link margin calculated from FSO link parameters are used for calculating the link availability. @ 2009 Society of Photo-Optical Instrumentation Engineers. [DOI: 10.1117/1.3155431]
\end{abstract}

Subject terms: free space optics; link budget; atmospheric attenuation; link availability; meteorological visibility; scattering; scintillation.

Paper 080912R received Nov. 21, 2008; revised manuscript received Apr. 15, 2009; accepted for publication Apr. 16, 2009; published online Jun. 23, 2009.

\section{Introduction}

The free space optics (FSO) communication is a recent and growing technology that has found application in many areas of the short- and long-haul communications space (from intersatellite links to interbuilding links). The terrestrial FSO systems combine some advantageous capabilities of fiber optics (high data rates, no mutual interference between the FSO systems, and difficult eavesdropping on transmitted data), and radio frequency equipment (wireless connectivity, fast and easy installation, and relatively low cost).

A disadvantage of FSO is a fluctuation of atmospheric attenuation caused by a number of phenomena in the atmosphere, such as scattering, absorption, and turbulence. Scattering in particular, which is a product of fog, haze, or low clouds, causes large variation in the received optical power and markedly limits the availability of FSO for a given transmission range.

In terrestrial applications, the FSO systems are most frequently used as the last-mile telecommunications link or as the LAN link between buildings. For telecommunication (carrier-class) applications, the link availability is generally considered to be $99.999 \%$ while for the LAN applications (enterprise-class) a link availability of over $99 \%$ is usually sufficient. ${ }^{-1}$

The availability calculation in the present work is based on the power budget analysis of FSO and on the statistical analysis of atmospheric attenuation. Power loss due to scattering is calculated using visibility data collected during several years at a few airports in Europe, and power loss caused by turbulence is modeled using the Rytov scintilla-

0091-3286/2009/\$25.00 @ 2009 SPIE tion theory ${ }^{2}$. The availability is defined as a time in which the atmospheric attenuation is lower than the power margin of FSO [i.e., data are transferred at a given or lower bit error rate (BER)] related usually to one or more years.

A beam with an ideal Gaussian intensity profile corresponding to the theoretical TEM00 mode is the most frequently used model for the real laser-beam description. But most FSO systems use large divergence beams of the order of milliradians to allow easy alignment. In the case of relatively large divergence angles and relatively large link distances, this model can be considerably simplified without any notable loss of accuracy.

Because, due to rounding to hundreds of meters, the collected visibility data are inaccurate and the strength of turbulence is hard to estimate correctly (especially in fog or rain), the eventual inaccuracy caused by simplifying the Gaussian beam model or by using the spherical wave model instead of the exact Gaussian beam model is negligible.

\section{FSO Link Budget}

An example of the power level diagram of FSO deployed at a distance of $\sim 1 \mathrm{~km}$ is shown in Fig. 1. The intervals between the two neighboring points on the horizontal axis represent specific parts of FSO causing particular attenuation observable between a laser diode at one side and a photodiode at the other side of FSO.

It is obvious that the received optical power can be written in the form

$P_{4}=P_{0}-A_{\mathrm{tx}}-A_{\text {prop }}-A_{\mathrm{atm}}-A_{\mathrm{rx}} \quad[\mathrm{dBm}]$,

where $P_{0}$ is the mean optical power of a laser diode, $A_{\mathrm{tx}}$ includes the coupling loss between the laser and the trans- 


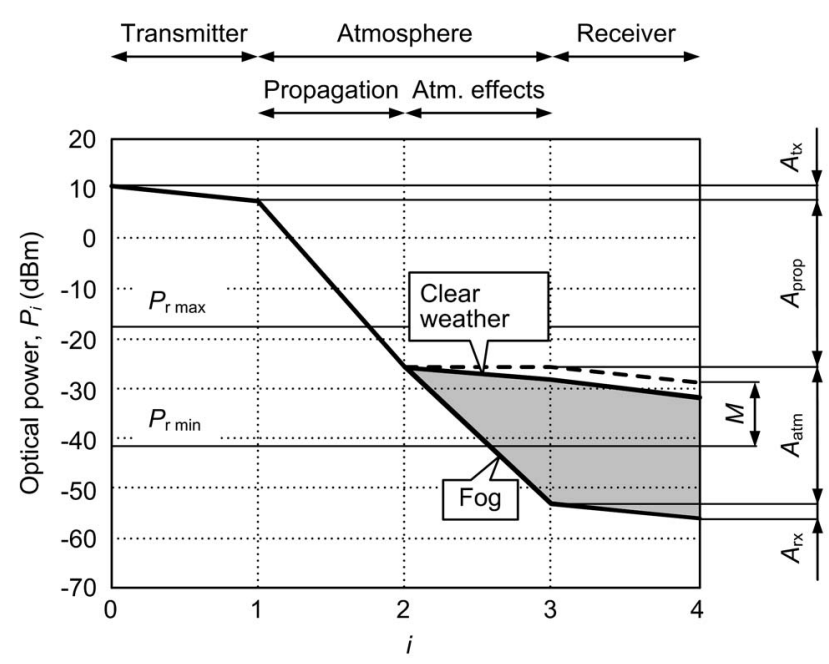

Fig. 1 An example of the FSO power level diagram.

mitter lens and the attenuation loss in the lens, $A_{\text {prop }}$ is the beam attenuation due to propagation loss, $A_{\text {atm }}$ includes random losses caused by atmospheric phenomena (scattering and turbulence), and $A_{\mathrm{rx}}$ represents the coupling loss between the receiver lens and photodiode and the attenuation and reflection at the lens.

For a successful detection, the power on the active area of the photodiode $P_{4}$ has to be inside the interval bounded by the receiver sensitivity $P_{\mathrm{r} \text { min }}$ and by the receiver saturation $P_{\mathrm{r} \text { max }}$ (minimum and maximum received optical power for the given BER). It is obvious that in the example given this requirement is not fulfilled for fog.

\section{Propagation Loss}

The Gaussian beam that propagates along the $z$-axis can be characterized at the transmitter $(z=0)$ by the beam spot radius $W_{0}$, at which the optical intensity falls off to $1 / e^{2}$ of the maximum on the beam axis, and by the radius of curvature $F_{0}$, which specifies the forming of the beam. The cases $F_{0}=\infty, F_{0}>0$, and $F_{0}<0$ correspond to collimated, convergent, and divergent beam forms, respectively. These parameters are usually used to describe the beam at a given position $z=L$ by the so-called input-plane beam parameters $^{2,3}$

$\Theta_{0}=1-\frac{L}{F_{0}}, \quad \Lambda_{0}=\frac{2 L}{k W_{0}^{2}}$,

where symbol $\Theta_{0}$ denotes the curvature parameter, $\Lambda_{0}$ is the Fresnel ratio at the input plane, $k=2 \pi / \lambda$ is the wave number, and $\lambda$ is the wavelength.

An additional parameter useful for beam description is the divergence half-angle, which defines the spreading of the beam when propagating toward infinity. It is given by

$\theta=\frac{\lambda}{\pi W_{\mathrm{B}}}$

where
$W_{\mathrm{B}}=\frac{W_{0}}{\left[\left(k W_{0}^{2} / 2 F_{0}\right)^{2}+1\right]^{1 / 2}}$

is the spot size radius at the beam waist (i.e., at the minimum beam radius along the path).

The optical intensity in the Gaussian beam at radial distance $r$ from the optical axis is ${ }^{2}$

$I(r, L)=I_{0} \frac{W_{0}^{2}}{W^{2}(L)} \exp \left[-\frac{2 r^{2}}{W^{2}(L)}\right]$,

where $I_{0}=I(0,0)$ is the transmitter output intensity at the centerline of the beam, and $W(L)$ is the beam spot radius given by

$W(L)=W_{0}\left[\Theta_{0}^{2}(L)+\Lambda_{0}^{2}(L)\right]^{1 / 2}$.

Because the peak optical intensity in the Gaussian beam is twice the average intensity ${ }^{4}$ and the total power is equal to the average intensity multiplied by the beam spot area, the relation between the intensity of the optical wave and the total power in the beam $P_{0}$ for the case $r=0$ is

$I(0, L)=I_{0} \frac{W_{0}^{2}}{W^{2}(L)}=\frac{2 P_{0}}{\pi W^{2}(L)}$.

Power $P$ incident on the circular receiver lens of aperture diameter $D$ situated at distance $L$ can be calculated according to Ref. 4 as

$P(D, L)=P_{0}\left[1-\exp \left(-\frac{D^{2}}{2 W^{2}(L)}\right)\right]$.

In a real situation, a large divergence angle $(\theta \geqslant 1 \mathrm{mrad})$ and relatively large beam spot radius $\left(W_{0} \geqslant 10 \mathrm{~mm}\right)$ cause that $k W_{0}^{2} / 2 F_{0} \gg 1$, and the divergence calculation using Eqs. (3) and (4) reduces to $\theta=W_{0} /\left|F_{0}\right|$. Similarly, because $\Theta_{0} \gg \Lambda_{0}$, Eq. (6) can be expressed in simplified form as $W(L)=W_{0}+L \theta$. On the assumption that the beam radius at the receiver position is much greater than the diameter of the receiver lens (i.e., $W(L) \gg D$ ), the optical intensity at the lens may be regarded as uniformly distributed and the received power can be expressed in the simple form

$P(D, L)=I(0, L) \frac{\pi D^{2}}{4}$.

Substituting Eq. (7) into Eq. (9), the propagation loss may be expressed as

$$
\begin{aligned}
A_{\text {prop }}(D, L) & =10 \log \left[\frac{P_{0}}{P(D, L)}\right] \\
& =20 \log \left[\frac{\sqrt{2}\left(W_{0}+L \theta\right)}{D}\right] \quad[\mathrm{dB}] .
\end{aligned}
$$

\section{FSO Power Link Margin}

The laser power, beam divergence, receiver sensitivity, coupling losses, and receiver lens area define how the FSO is able to eliminate atmospheric effects. They are summarized 
Table 1 FSO system parameters used in calculations.

\begin{tabular}{lccc}
\hline \hline & FSO A & FSO B & FSO C \\
\hline$P_{0}[\mathrm{dBm}]$ & 10 & 13 & 13 \\
$P_{\mathrm{r} \min }[\mathrm{dBm}]$ & -36 & -39 & -39 \\
$A_{\mathrm{tx}}+A_{\mathrm{rx}}[\mathrm{dB}]$ & 4 & 6 & 6 \\
$W_{0}[\mathrm{~mm}]$ & 20 & 20 & 20 \\
$F_{0}[\mathrm{~m}]$ & -10 & -10 & -16 \\
$2 \theta[\mathrm{mrad}]$ & 4 & 4 & 2.5 \\
$D[\mathrm{~mm}]$ & 70 & 140 & 280 \\
$M_{0}[\mathrm{~dB}]$ & 70 & 80 & 90 \\
\hline \hline
\end{tabular}

in the power link margin $M$ (see Fig. 1). Taking into consideration the fact that the condition $W_{0} \ll L \theta$ is in a real situation usually fulfilled, the power link margin expressed in the simplified form resulting from Eq. (10) is given by

$M(L)=P_{0}-A_{\mathrm{tx}}-20 \log \left(\frac{\sqrt{2} L \theta}{D}\right)-A_{\mathrm{rx}}-P_{\mathrm{r} \min }[\mathrm{dB}]$

or by $M(L)=M_{0}-20 \log L$, where $M_{0}$ includes all constant values in Eq. (11) given by the FSO design.

By comparing real FSO systems designed for certain transmission ranges and data rates produced by various manufacturers, it can be found that the particular parameters (laser power, beam divergence, receiver sensitivity, etc.) of these systems are in some cases markedly different. Because of this fact, it is not easy to create a detailed FSO model characterizing a specific group of FSO systems. Fortunately, as shown below, to demonstrate the effects of turbulence and scattering on laser-beam propagation the FSO systems may be characterized only by the receiver lens area and by the power link margin $M_{0}$ or $M(L)$, respectively. Therefore, three typical FSO representatives, differing in the power link margin and in the diameter of the circular receiver lens, were created for simulation. Their particular parameters, summarized in Table 1, were chosen such that they characterize typical real systems designed for a data rate of $1 \mathrm{Gbps}$ and for three different operating ranges (see Section 5). A real FSO system whose particular parameters are measurably different can, for the link availability evaluation, be assigned to the appropriate FSO representative on the basis of calculated $M_{0}$ or possibly on the basis of calculated or measured $M(L)$. The power link margins $M(L)$ for FSO representatives, calculated according to approximate relation (11) and according to an exact relation based on Eq. (8), are shown in Fig. 2 (solid and dashed lines).

It can be deduced that, at very short link distances, the power link margin is not affected by the propagation loss because the beam spot diameter at the receiver position is lower than the diameter of the receiver lens. Thus, the entire power in the beam is received. A growing link distance causes that the Gaussian intensity distribution at the receiver lens increasingly resembles uniform distribution and

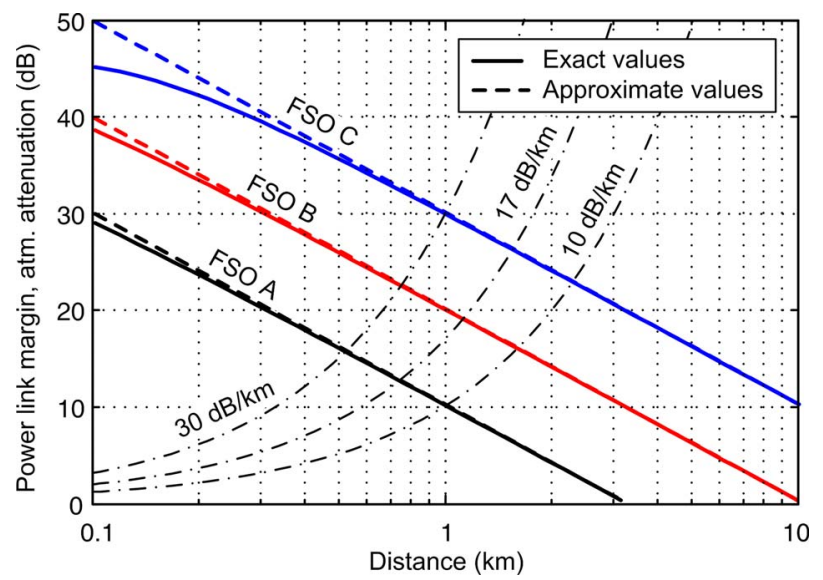

Fig. 2 Power link margin and specific atmospheric attenuation versus link distance.

that the results of exact calculation (based on Gaussian distribution) and approximate calculation (based on uniform distribution) converge.

\section{Attenuation Due to Scattering}

Attenuation in atmosphere is inversely proportional to the transmittance, which is described by the Beers-Lambert law $^{5}$

$T(L)=\frac{I(L)}{I(0)}=\exp \left(-\alpha_{e} L\right)$,

where $I(0)$ and $I(L)$ are the optical intensities at the source and at a distance $L$, respectively, and $\alpha_{e}$ is the atmospheric attenuation coefficient. It can be expressed in terms of meteorological visibility $V$, defined as a distance at which transmittance falls to a certain value $\varepsilon$ [i.e., $T(V)=\varepsilon$ ]. In 1924 , Koschmieder, on the basis of intuitive evaluation of the contrast threshold, proposed the value 0.02 for $\varepsilon .{ }^{6}$ To meet aeronautical requirements, the World Meteorological Organization later adopted the value 0.05 because it ensured the requirement for reliably resolving a black object against the horizon in daylight at a wavelength of $550 \mathrm{~nm}$, where the human eye has the highest sensitivity. Assuming that $\varepsilon=0.05$, the atmospheric attenuation coefficient is

$\alpha_{e}=\frac{-\ln (0.05)}{V}=\frac{3}{V}$.

Atmospheric attenuation expressed in the decibel scale is related to transmittance by

$A_{10}(L, V)=-10 \log _{10}[T(L, V)]=10 \log _{10}(e) \alpha_{e}(V) L$,

and hence, the atmospheric attenuation coefficient in the decibel per unit length is approximately

$\alpha_{10}=4.343 \alpha_{e}$.

The predominant phenomenon causing light attenuation in fog is the Mie scattering. ${ }^{7,8}$ The atmospheric attenuation coefficient for attenuation due to scattering can be derived from Eqs. (13) and (15), taking into consideration the 
wavelength dependence of $\alpha_{e}$. For the case of wavelengths in the range from the visible to the near-infrared light and visibility given in kilometers it can be calculated according to the semi-empirical Kruse formula ${ }^{7}$ modified for $\varepsilon=0.05$ as follows

$\alpha_{10, \text { scat }}(V)=\frac{13}{V}\left(\frac{\lambda \times 10^{9}}{550}\right)^{-q(V)}[\mathrm{dB} / \mathrm{km}]$,

where $q(V)$ is the particle size distribution coefficient defined as

$q(V)= \begin{cases}1.6 & \text { for } V>50 \mathrm{~km} \\ 1.3 & \text { for } 6<V<50 \mathrm{~km} \\ 0.585 V^{1 / 3} & \text { for } 0<V<6 \mathrm{~km}\end{cases}$

The attenuation caused by scattering in decibel scale $A_{10 \text {,scat }}$ is in compliance with Eq. (14) given by the product of the atmospheric attenuation coefficient $\alpha_{10 \text {,scat }}$ and the link distance (in kilometers).

The latest investigations indicate no wavelength dependence of the atmospheric attenuation coefficient in foggy conditions, where the visibility is $<500 \mathrm{~m}$. A new method for evaluating the particle size distribution coefficient that respects this fact was proposed by Kim et al. ${ }^{8}$

$q(V)= \begin{cases}1.6 & \text { for } V>50 \mathrm{~km} \\ 1.3 & \text { for } 6<V<50 \mathrm{~km} \\ 0.16 V+0.34 & \text { for } 1<V<6 \mathrm{~km} \\ V-0.5 & \text { for } 0.5<V<1 \mathrm{~km} \\ 0 & \text { for } V<0.5 \mathrm{~km} .\end{cases}$

Further studies of attenuation due to scattering were conducted by a number of other authors. Naboulsi proposed relations for attenuation caused by radiation and advection fog for wavelengths from 690 to $1550 \mathrm{~nm}$ and for visibilities ranging from 50 to $1000 \mathrm{~m} .^{9,10}$

Radiation fog generally forms during the night when the temperature of the ground surface drops due to the radiation of the heat accumulated during the day. When the air is cooled by the ground surface below the dew point, the condensation of water vapor and, consequently, the formation of ground fog occur. The attenuation coefficient for radiation fog is

$\alpha_{10, \text { scat }}(V)=4.343 \frac{0.11478 \lambda_{\mu}+3.8367}{V}[\mathrm{~dB} / \mathrm{km}]$,

where $\lambda_{\mu}$ is the wavelength in microns.

Advection fog is formed when the warm and wet air moves above colder maritime or terrestrial (e.g., snow covered) surfaces. As in the previous case, the air in contact with the ground surface can be cooled below the dew point, which causes the condensation of water vapor. The attenuation coefficient for advection fog is given by

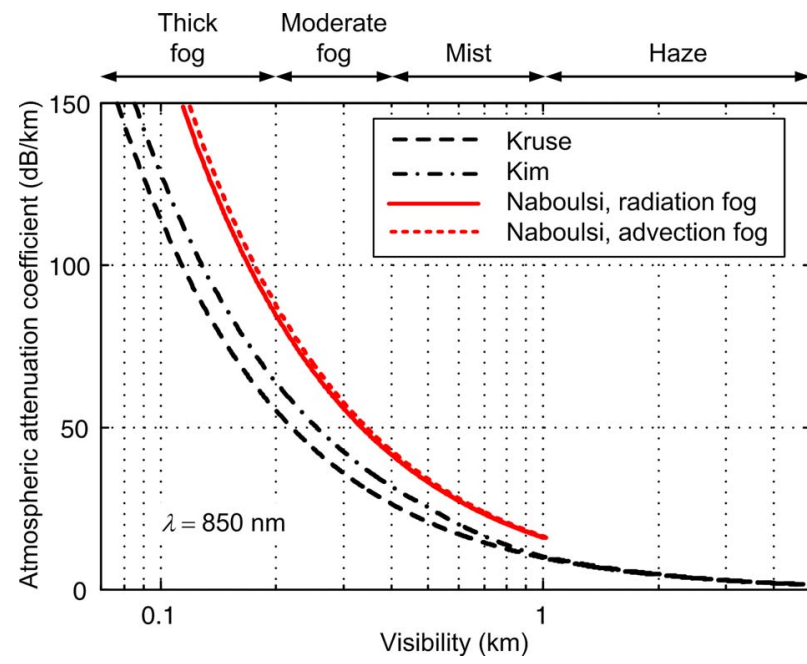

Fig. 3 Comparison of the formulas used for calculating the attenuation due to scattering.

$$
\begin{aligned}
& \alpha_{10, \text { scat }}(V) \\
& \quad=4.343 \frac{0.18126 \lambda_{\mu}^{2}+0.13709 \lambda_{\mu}+3.7205}{V}[\mathrm{~dB} / \mathrm{km}] .
\end{aligned}
$$

A graphical interpretation of the above formulas expressing attenuation due to scattering is shown in Fig. 3. The classification of fog, mist, and haze in relation to the visibility corresponds to the classification presented in Ref. 8. All calculations presented below were performed for a wavelength of $850 \mathrm{~nm}$. It is evident that the two Naboulsi formulas exhibit higher atmospheric attenuation compared to the Kim relation and, hence, they seem to be derived for $\varepsilon=0.02$.

Other sources of atmospheric attenuation (rain or snow) are discussed, for example, in Refs. 11 and 12. According to Ref. 12, the Kim relations (16) and (18) can be used with sufficient accuracy for the approximation of attenuation due to wet snow.

Some manufacturers of FSO systems give among technical parameters of their products, the recommended operating ranges defined usually for moderate fog $\left(\alpha_{10} \approx 30 \mathrm{~dB} / \mathrm{km}\right)$, medium snow, cloud-burst or mist $\left(\alpha_{10} \approx 17 \mathrm{~dB} / \mathrm{km}\right)$, and for medium to heavy rain, light snow, or haze $\left(\alpha_{10} \approx 10 \mathrm{~dB} / \mathrm{km}\right)$. It can be seen from the atmospheric attenuations $\alpha_{10} L$, plotted in Fig. 2 (dashed and dotted lines) that, for example, the recommended range in moderate fog is $520 \mathrm{~m}$ for FSO A, $750 \mathrm{~m}$ for FSO B, and $1000 \mathrm{~m}$ for FSO C. The recommended operating ranges can be used to choose an appropriate FSO representative.

\section{Power Loss in Turbulence}

The most important effects of atmospheric turbulence on the laser beam are phase-front distortion, beam broadening, beam wander, and redistribution of intensity within the beam, known as scintillation. In the case of strongly divergent beam, scintillation is the most significant source causing a loss of power. 
Evaluating the power losses due to turbulence is a marginal problem in the availability calculation in this paper. Because of the absence of real data, it is very difficult to estimate scintillation strengths in different weather conditions and to determine the effect of scintillation on longterm FSO availability. The aim of this section is only to give relations for a rough estimation of the power loss in turbulent atmosphere. That is why some simplifications have been made: power loss was solved for the weak fluctuation regime, the laser beam characteristics were approximated by the spherical wave, and the power loss conditional on a maximum probability of having a BER below a certain value was used. ${ }^{13}$

The intensity $I$ of an optical wave propagating through turbulent atmosphere is a random variable. The normalized variance of optical wave intensity, referred to as the scintillation index, is defined by ${ }^{2}$

$\sigma_{I}^{2}=\frac{\left\langle I^{2}\right\rangle}{\langle I\rangle^{2}}-1$

where the angular brackets denote an ensemble average. The scintillation index indicates the strength of intensity fluctuations. For weak fluctuations, it is proportional and, for strong fluctuations, it is inversely proportional to the Rytov variance for a plane wave

$\sigma_{1}^{2}=1.23 C_{n}^{2} k^{7 / 6} L^{11 / 6}$,

where $C_{n}^{2}$ is the refractive-index structure parameter. ${ }^{2}$ This parameter is not easy to measure because it depends on the temperature, wind strength, altitude, humidity, atmospheric pressure, etc. It varies approximately from $10^{-12}$ to $10^{-19} \mathrm{~m}^{-2 / 3}$. Some methods leading to its evaluation are presented in Refs. 14-16. For a homogenous turbulent field, which can be assumed for near-ground horizontal-path propagation, the refractive-index structure parameter is constant.

Optical scintillations can be reduced by increasing the collection area of the receiver lens; this area causes an integration of various intensities incident on particular parts of the lens. This phenomenon is known as aperture averaging. The aperture averaging factor for a spherical wave is

$A=\frac{\sigma_{P}^{2}}{\sigma_{I}^{2}}=\left[1+0.333\left(\frac{k D^{2}}{4 L}\right)^{5 / 6}\right]^{-7 / 5}$,

where $\sigma_{P}^{2}$ is the power scintillation index and $\sigma_{I}^{2}=0.4 \sigma_{1}^{2}$ is the Rytov variance for the spherical wave. ${ }^{17}$ The effect of the aperture averaging factor on the power scintillation index is demonstrated in Fig. 4, where two different refractive-index structure parameters were used for the calculation. The receiver lens diameters can be found in Table 1. It can be observed that the influence of both the lens diameter and the refractive-index structure parameter on the scintillation level is relatively large.

Many theoretical and experimental works (see, for example, Refs. 2, 17, and 18) show that under weak fluctuations, the optical power or intensity may be modeled as a lognormally distributed random variable. The probability density function (PDF) of the received optical power is then of the form

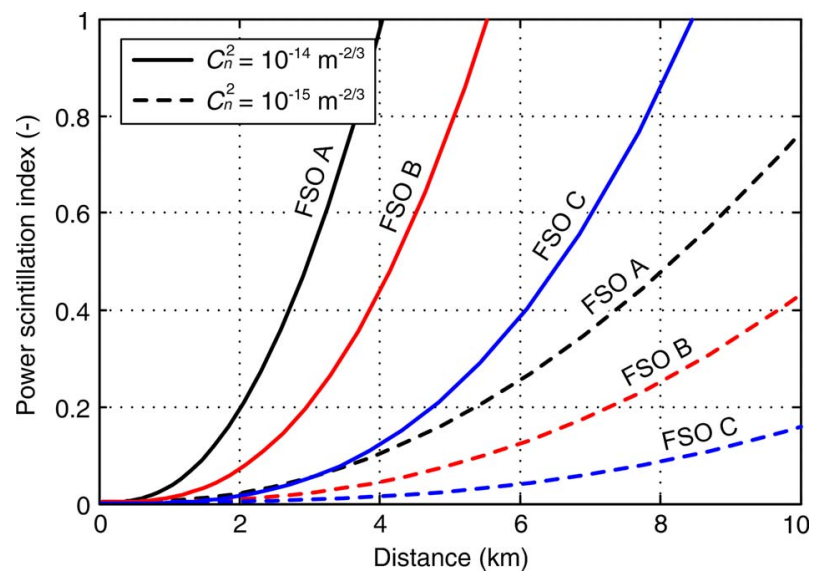

Fig. 4 Power scintillation index at FSO receivers versus link distance.

$p(P)=\frac{1}{P \sqrt{2 \pi \sigma^{2}}} \exp \left\{-\frac{\left[\ln (P /\langle P\rangle)+\sigma^{2} / 2\right]^{2}}{2 \sigma^{2}}\right\}$,

where $\sigma^{2}=\ln \left(\sigma_{P}^{2}+1\right)$ is the variance of the log power.

The bit error rate obtained during the communication through the turbulent-free atmosphere is lower than the bit error rate affected by scintillation (i.e., $\mathrm{BER}_{0}<\mathrm{BER}_{\mathrm{sc}}$ ). This is caused by the power fluctuations, which can result in momentary fades of signal. It is evident that an increase of $\mathrm{BER}_{\mathrm{sc}}$ can be reduced by a corresponding increase of optical power.

One of a few known ways leading to the definition of losses due to scintillation $L_{\mathrm{sc}}$ is based on the assumption that the probability of the inequality $\mathrm{BER}_{0}<\mathrm{BER}_{\mathrm{sc}}$ is kept at a certain value, i.e.,

$\operatorname{Pr}\left[\mathrm{BER}_{0}<\mathrm{BER}_{\mathrm{sc}}\right]=\operatorname{Pr}\left[P L_{\mathrm{sc}}<\langle P\rangle\right]=P_{\mathrm{ub}}$,

where $P L_{\mathrm{sc}}$ is the compensated instantaneous power, which is, in general, lower than the average power $\langle P\rangle$ within the time intervals whose sum is equal to the upper bound probability $P_{\text {ub }}$ multiplied by the transfer time. Because scintillation is a momentary redistribution of intensity without loss of power and the beam broadening due to turbulence is negligible for large divergence angles, the average received power $\langle P\rangle$ equals $P(D, L)$ given by Eq. (9).

By rearranging Eq. (25) and assuming that $P$ is lognormally distributed according to Eq. (24), we obtain

$\operatorname{Pr}\left[\frac{P}{\langle P\rangle}<\frac{1}{L_{\mathrm{sc}}}\right]=F\left(\frac{1}{L_{\mathrm{sc}}}\right)=\frac{1}{2} \operatorname{erfc}\left[-\frac{\ln \left(1 / L_{\mathrm{sc}}\right)+\sigma^{2} / 2}{\sqrt{2 \sigma^{2}}}\right]$,

where $F($.$) is the cumulative distribution function (CDF)$ and $\operatorname{erfc}($.$) is the complementary error function. Hence, the$ scintillation loss is of the form

$L_{\mathrm{sc}}=\exp \left[\operatorname{erfcinv}\left(2 P_{\mathrm{ub}}\right) \sqrt{2 \ln \left(\sigma_{P}^{2}+1\right)}\right] \sqrt{\sigma_{P}^{2}+1}$,

where erfcinv(.) is the inverse complementary error function. Because $\sigma_{P}$ is a function of $L$, we can finally write 


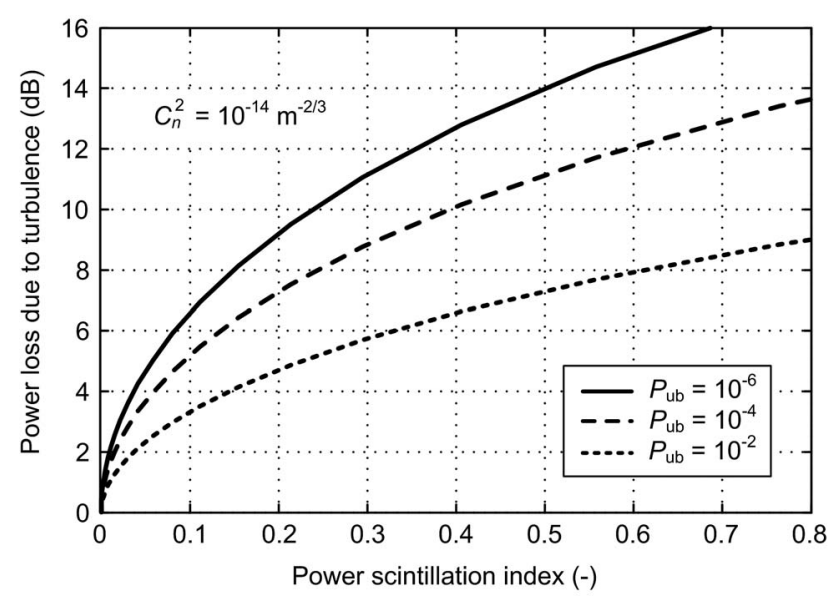

Fig. 5 Power loss caused by turbulence plotted for differing upper bound probabilities.

$A_{\text {turb }}(L)=10 \log \left[L_{\mathrm{sc}}(L)\right] \quad[\mathrm{dB}]$.

Examples of power loss caused by turbulence differing in the upper bound probability are shown in Fig. 5. The link distance at which the power link margin equals the power loss caused by turbulence can be found in Fig. 6 for all three representatives of FSO. Calculation was performed for the refractive-index structure parameter of $10^{-14} \mathrm{~m}^{-2 / 3}$. Any FSO system deployed at a link distance longer than the distance given by the corresponding point of intersection will exhibit a probability of $\mathrm{BER}_{0}<\mathrm{BER}_{\mathrm{sc}}$ of $>10^{-4}$.

\section{Link Availability}

A correct operation of the FSO link will be achieved if the condition

$M(L) \geqslant A_{\mathrm{atm}}(L)$

is true for the required link distance $L$. As mentioned above, atmospheric attenuation is generally composed of power loss due to scattering and power loss due to turbu-

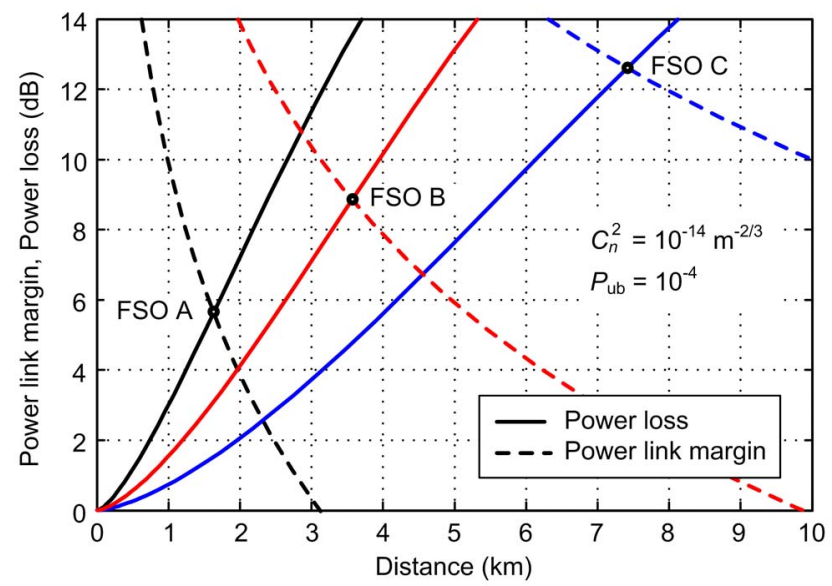

Fig. 6 Power link margin and power loss caused by turbulence, calculated for all three FSO representatives.

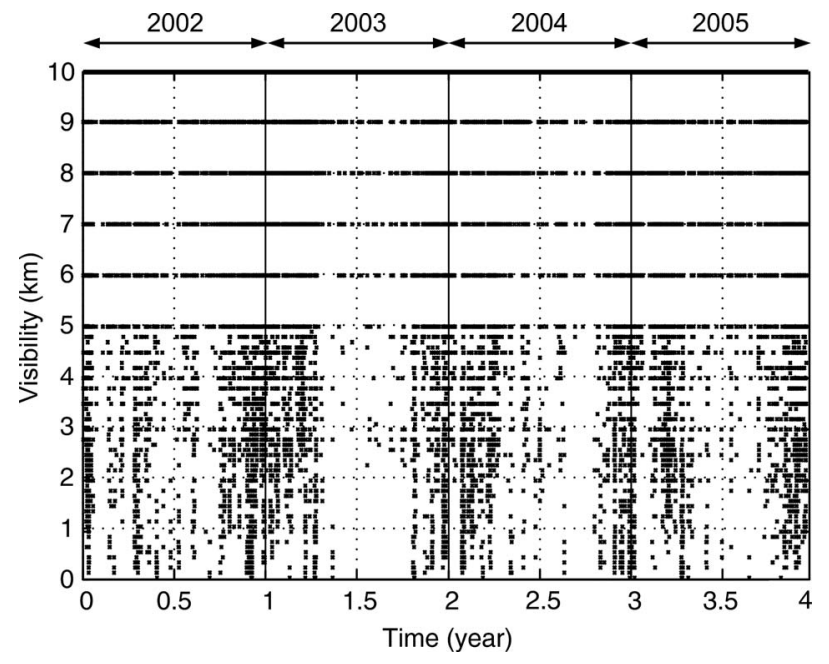

Fig. 7 Visibility data collected at the Schoenefeld International Airport in Berlin (EDDB) from January 1, 2002 to December 31, 2005.

lence. For the link availability calculation, the statistical data of both types of power loss collected during the relatively long period (longer than one year) are needed. Although these data are not available, only attenuation due to scattering was calculated from the collected visibility data using Eq. (14) according to

$A_{\text {atm }}(L, V)=A_{10, \text { scat }}(L, V)=\alpha_{10, \text { scat }}(V) L \quad[\mathrm{~dB}]$.

The visibility data are available from reports of selected airports in Germany, France, and Italy for the years 2002 to 2005. The sampling period of the visibility measurement is $30 \mathrm{~min}$, and the visibility resolution is $100 \mathrm{~m}$ in the range from $100 \mathrm{~m}$ to $5 \mathrm{~km}$, and $1 \mathrm{~km}$ in the range from 5 to $10 \mathrm{~km}$. The visibility was measured as the runway visual range (RVR). The data collected by an RVR measuring device (transmissometers or forward scatter visibility sensors) is an average value, with averaging intervals much longer than the duration of fades caused by turbulence, and therefore, turbulence does not affect the result of measurement. An example of the data collected at the Schoenefeld International Airport in Berlin, whose International Civil Aviation Organization (ICAO) code is EDDB, from January 1, 2002 to December 31, 2005 is shown in Fig. 7. The periodical changing of seasons is noticeable in Fig. 7. PDF and CDF calculated from the same data and CDF approximated by a polynomial of degree 8 using the least-squares method are depicted in Fig. 8. From CDF, it can be seen, for example, that atmospheric visibility in the range measured by the RVR measuring devices $(0 \leqslant V<10 \mathrm{~km})$ occurs with a probability of 0.2 .

Substituting Eqs. (16) and (30) into Eq. (29) and then solving Eq. (29) for $V$ provided that the link distance is expressed in kilometers, we obtain

$V \geqslant \frac{13 L}{M(L)}\left(\frac{\lambda \times 10^{9}}{550}\right)^{-q(V)} \quad[\mathrm{km}]$.

The right-hand side of Eq. (31) represents the minimal required visibility for a correct operation of FSO (note that it also depends on $V$ ). Inequality (31) can then be written in 


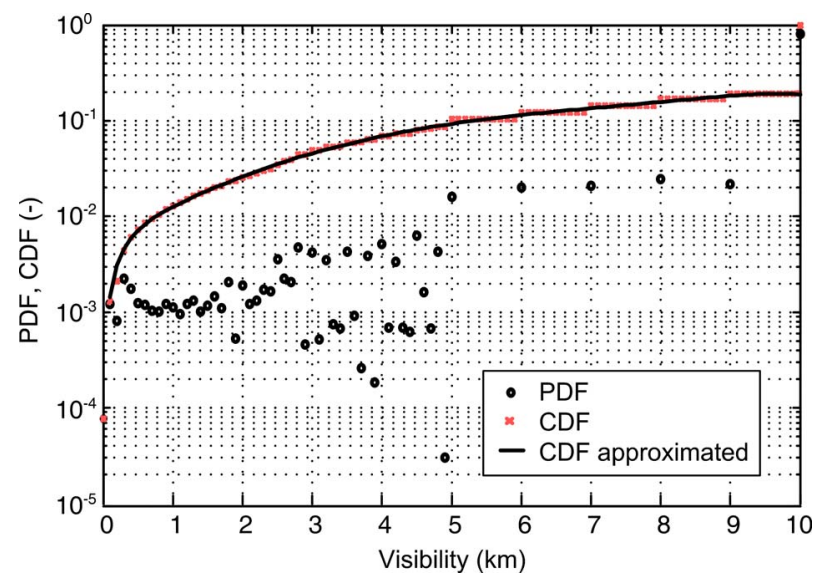

Fig. 8 PDF, CDF, and approximated CDF calculated from the data collected at the Schoenefeld International Airport in Berlin between the years 2002 and 2005 in dependence on visibility.

the simple form $V \geqslant V_{\min }(L, V)$. Solving (31) numerically with the equality sign yields the values $V_{\min }$ for the given $L$. Similar inequality can be obtained in the same way for the Naboulsi relation (19)

$V \geqslant 4.343 \frac{0.11478 \lambda_{\mu}+3.8367}{M(L)} L[\mathrm{~km}]$.

The minimum required visibilities, calculated according to the Kim and the Naboulsi relations for the A, B, and C links deployed at distances from $100 \mathrm{~m}$ to $4 \mathrm{~km}$, are depicted in Fig. 9.

Considering the visibility $V$ a random variable, the FSO link availability is defined as

$L_{\mathrm{A}}=\operatorname{Pr}\left[V \geqslant V_{\min }(L)\right]=1-F\left[V_{\min }(L)\right]$.

The link availabilities calculated from visibility data collected at the Schoenefeld International Airport in Berlin according to Eqs. (31)-(33), where $F\left[V=V_{\min }(L)\right]$ is the CDF shown in Fig. 8, can for all three FSO representatives be seen in Fig. 10. Both the CDF calculated from visibility data and the CDF approximated by a polynomial of rela-

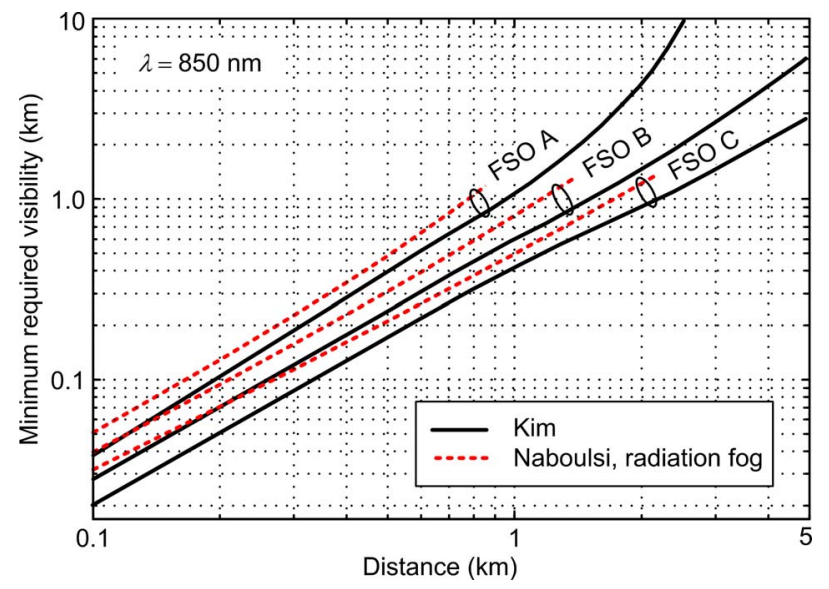

Fig. 9 Minimum required visibility for correct operation of FSO in dependence on link distance.

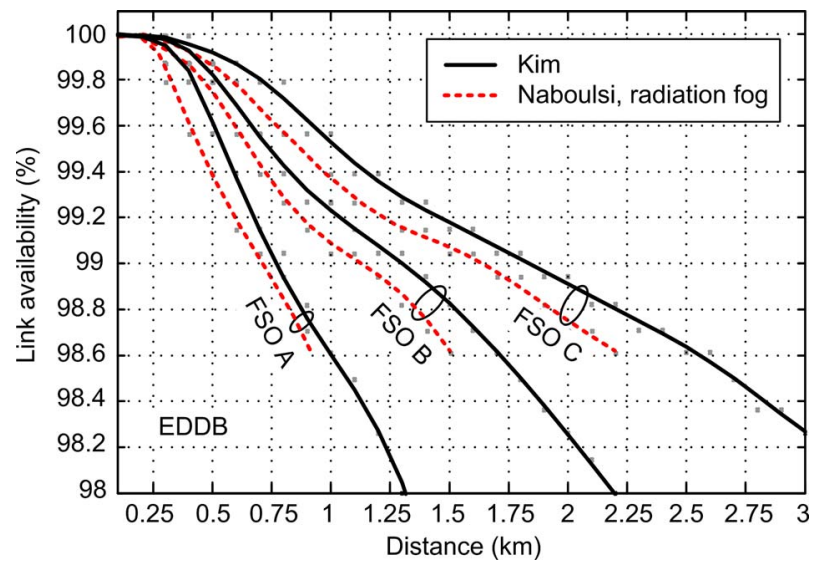

Fig. 10 Availability of FSO links in dependence on the link distance calculated from data collected at the Schoenefeld International Airport in Berlin.

tively high degree can be used for calculations without noticeable difference in results, but the availabilities obtained for short distances are only approximate. This is caused by the relatively rough rounding of the visibility measured.

It can be simply found that for a link availability of $99 \%$ the Kim relation gives an $\sim 1.15$ times longer minimum link distance than the Naboulsi relation does.

Examples of the FSO link availabilities calculated for the years 2002 to 2005 according to the Kim formula for selected airports in Germany, France, and Italy are shown in Figs. 11-14. The airports are identified by their ICAO codes. The names of airports are given only in case there are two airports in one city mentioned in this paper. The FSO B link was chosen for the calculations because it is a typical representative for a relatively large group of real FSO systems.

It is evident that the seaside areas in the south of Europe offer a higher FSO availability compared to the continental areas, due to the rare occurrence of fog. These areas exhibit a specific availability dependence on link distance, for

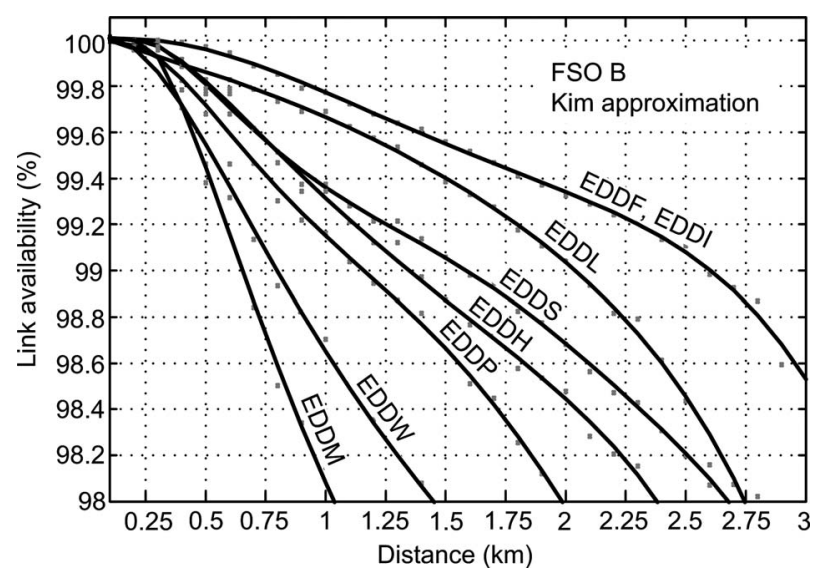

Fig. 11 Availability of FSO B link calculated from data collected at airports in Germany: Frankfurt (EDDF), Berlin-Tempelhof International Airport (EDDI), Duesseldorf International Airport (EDDL), Stuttgart (EDDS), Hamburg (EDDH), Leipzig/Halle (EDDP), Bremen (EDDW), and Munich (EDDM). 


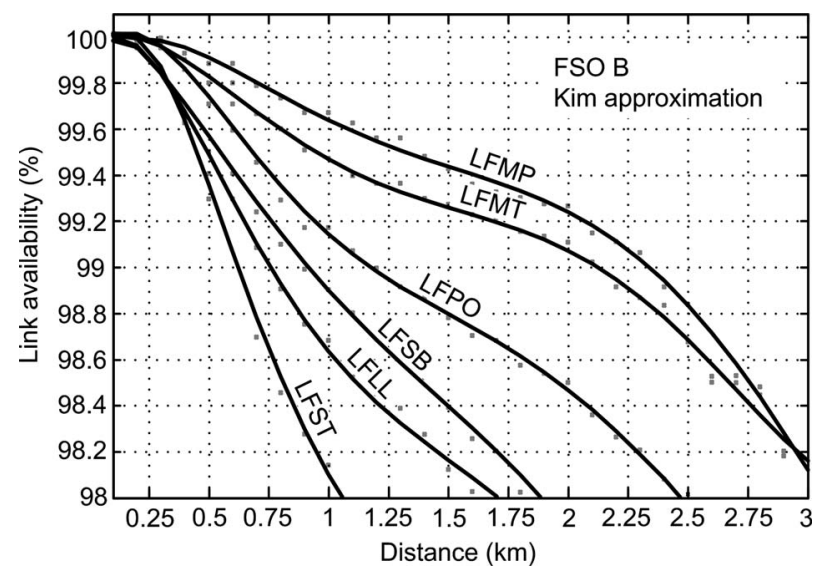

Fig. 12 Availability of FSO B link calculated from data collected at airports in France: Perpignan/Rivesaltes (LFMP), Montpellier (LFMT), Paris (LFPO), Basel/Mulhouse (LFSB), Lyon (LFLL), and Strasbourg (LFST).

which a relatively long sequence of almost constant values is typical, followed by a steep drop. An excellent FSO availability was obtained for Ajaccio (LFKJ) on Elba Island, Nice/Cote (LFMN), or Genoa (LIMJ). Very good link availability is in Palermo (LICJ) or Bari (LIBD). Similar results could be found, for example, for Marseille/Provence (LFML) or Toulon (LFTH). A relatively low availability was calculated for Strasbourg (LFST) but also for Munich (EDDM) or Milan/Bergamo (LIME). Another result, which was obtained for Hamburg (EDDH), is typical of many other cities, such as Berlin (EDDB), Paris (LFPO), Stuttgart (EDDS), Leipzig/Halle (EDDP), or other cities, not included in this paper, such as Grenoble (LFLS), Chambery (LFLB), Avignon (LFMV), Duesseldorf/ Moenchengladbach Airport (EDLN), or Dortmund (EDLW). It can be regarded as the Central European standard. A surprisingly good availability was obtained for Frankfurt (EDDF) and also for Tempelhof International Airport in Berlin (EDDI). The availability dependence obtained for the two airports is practically the same, and therefore, only one waveform was depicted in Fig. 11.

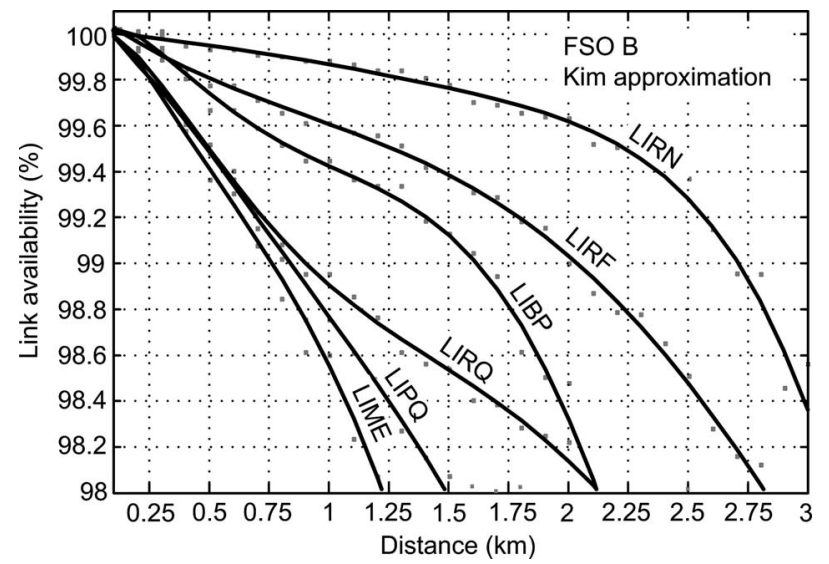

Fig. 13 Availability of FSO B link calculated from data collected at airports in Italy: Naples (LIRN), Rome (LIRF), Pescara (LIBP), Florence (LIRQ), Trieste (LIPQ), and Milan/Bergamo (LIME).

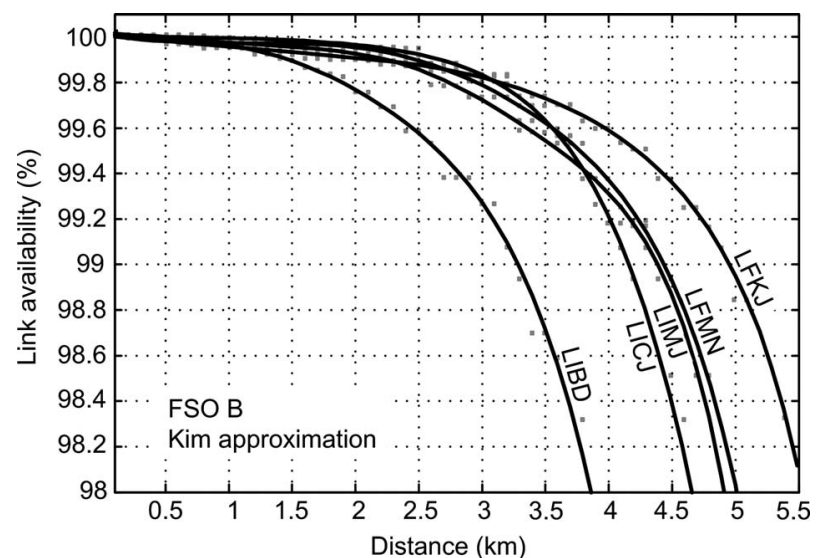

Fig. 14 Availability of FSO B link calculated from data collected at airports at the seaside of southern Europe: Ajaccio (LFKJ), Nice/ Cote (LFMN), Genoa (LIMJ), Palermo (LICJ), and Bari (LIBD).

All the FSO link availabilities shown in Figs. 10-14 were calculated from data collected within four or three years (some records are complete for only three years) and can be considered as long-term or average availabilities. However, the atmospheric conditions change from year to year and differ considerably according to the changing seasons. Examples of the link availabilities calculated for the particular years from the visibility records obtained at the Schoenefeld airport in Berlin (EDDB) and at Nice/Cote airport (LFMN) are shown in Figs. 15 and 16. On the basis of the results obtained, it can be concluded that the continental weather conditions cause evidently larger differences in link availability than the weather conditions in seaside areas in southern Europe.

The fact that the monthly obtained link availability differs in dependence on the year and on the locality of the visibility measurement is shown in Figs. 17 and 18, in which the availability is plotted for the particular months in the years 2003, 2004, and 2005 and for the four different airports. Although the most critical months for link availability evidently differ in particular calculations, the typical months that exhibit great link availability variations include January, March, November, and December.

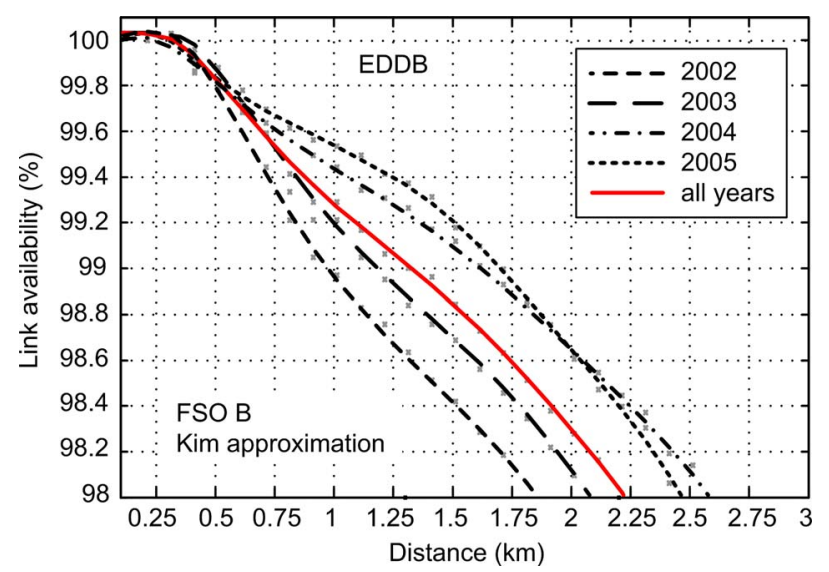

Fig. 15 Availability of FSO B link calculated for particular years from data collected at Schoenefeld airport in Berlin. 


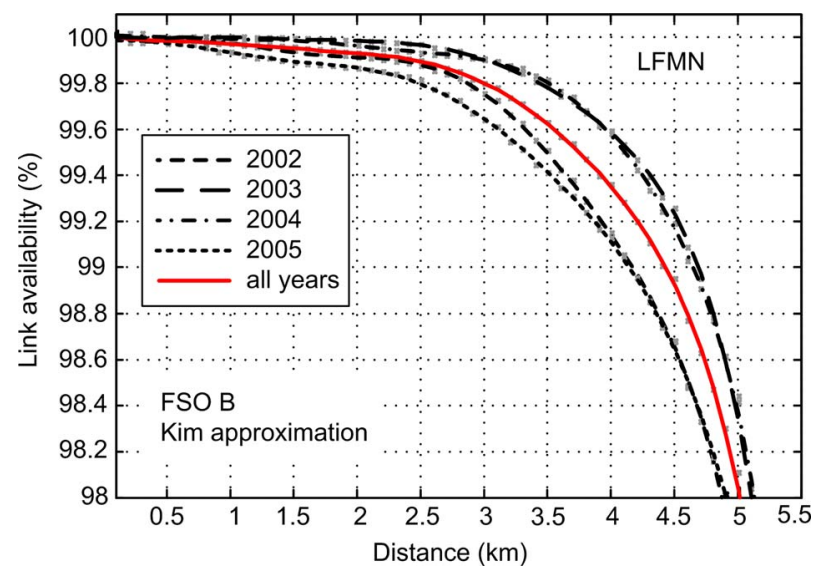

Fig. 16 Availability of FSO B link calculated for particular years from data collected at Nice/Cote airport.

It is obvious that the FSO systems designed for correct operation in foggy conditions need a large power link margin to overcome beam attenuation, which can reach tens of decibels per kilometer. Because the required power link margin can in many cases be ensured only by short link distances and the fade caused by scintillations appears only if the link distance is relatively large, these systems cannot be affected by turbulence. But, for example, the FSO B in the enterprise application, where the required link availability is $99 \%$, can be deployed in Palermo (LICJ) at a distance of $\sim 4.2 \mathrm{~km}$ (see Fig. 14). An admissible distance that avoids noticeable degradation of communication quality due to turbulence is $\sim 3.6 \mathrm{~km}$ (see Fig. 6). It is highly probable that in the case of stronger scintillations (higher $C_{n}^{2}$ ) the FSO availability will decrease due to turbulence and the scintillation effect has to be taken into consideration whenever a maximum usable link distance is being solved. Similar situations can arise also in other seaside areas in southern Europe. Note that at a distance of $\sim 3.6 \mathrm{~km}$ and at the refractive-index structure parameter of $10^{-14} \mathrm{~m}^{-2 / 3}$ scintil-

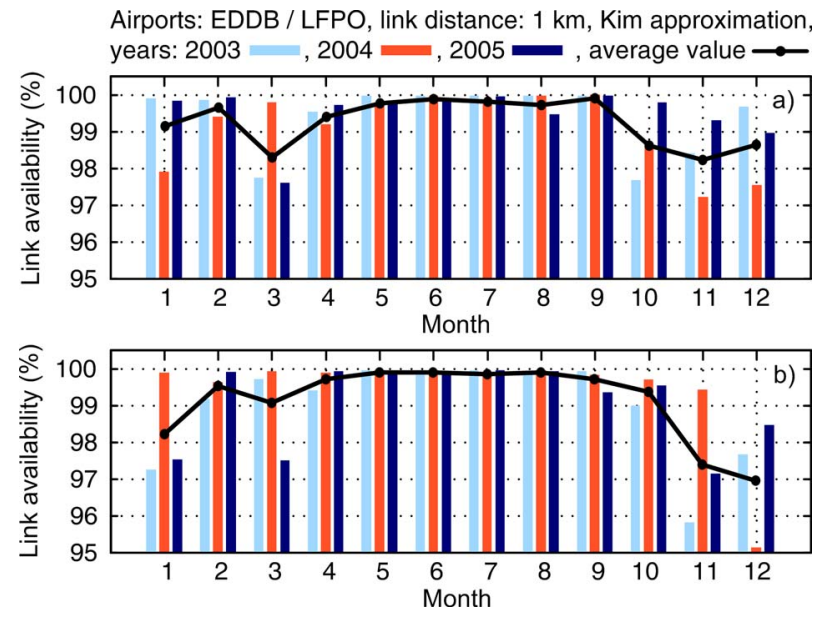

Fig. 17 Availability of FSO B link deployed at a distance of $1 \mathrm{~km}$ calculated for particular months and years from data collected at (a) Schoenefeld airport in Berlin and (b) at the airport in Paris.
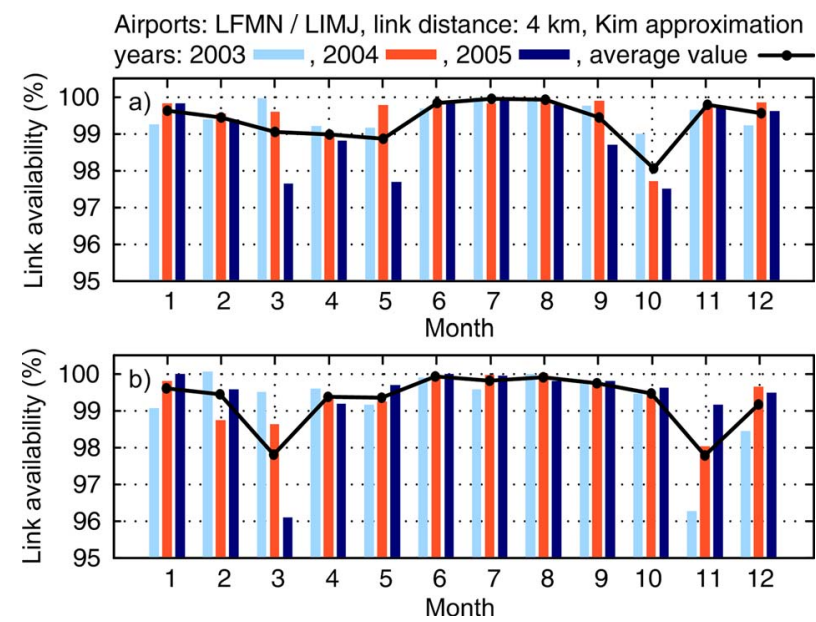

Fig. 18 Availability of FSO B link deployed at a distance of $4 \mathrm{~km}$ calculated for particular months and years from data collected at (a) Nice/Cote airport and (b) the airport in Genoa.

lation in reality reaches, already, the moderate (saturation) regime and the real the scintillation is slightly lower than the scintillation shown in Fig. $4 .^{17}$

Although the availability calculation was performed only for the FSO B link, the results can be adopted for the other FSO system availability estimation. In order to obtain an identical value of availability for any FSO link, the minimum visibility $V_{\min }\left(L_{x}\right)$ at link distance $L_{x}$ calculated for this link has to agree with $V_{\min }(L)$ calculated for FSO B [see Eqs. (31)-(33)]. The required link distance $L$ for a given FSO B availability can be found in Figs. 11-14 and the corresponding link distance $L_{x}$ for other FSO systems (FSO C, FSO A) can then be then read in Fig. 9, maintaining the minimum required visibility constant. The same procedure can be used for availability estimation of other FSO systems whose minimum required visibility in dependence on the link distance is known. The availability estimation procedure can be verified on the curves plotted in Fig. 10.

\section{Conclusion}

The aim of the paper has been to give a summary of calculation of power losses caused by turbulence and scattering, to compare the FSO availability in selected areas in Europe, and to show how this availability can be evaluated.

The FSO availability varies in dependence on power link margin and in dependence on the locality where the FSO is installed. It is possible to establish some rules for availability estimation that are of general validity; for example, good availability can be achieved in warm seaside areas or desert regions but, on the other hand, in large cities with two or more airports the availabilities obtained from these airports can differ considerably [e.g., Schoenefeld International Airport (EDDB) and Tempelhof International Airport (EDDI) in Berlin]. FSO availability at any location can thus be very difficult to reliably determine.

The data collected are suitable for evaluating the enterprise-class FSO appropriateness but not the carrierclass FSO appropriateness because of the low resolution of the visibility measured. However, it is evident that the 
availability required for the carrier-class systems can be achieved only for very short distances (a few hundred meters). Improved FSO availability can be obtained, for example, with the hybrid FSO/RF communication systems, where the RF link backs up (at lower data rates) the FSO if atmospheric attenuation exceeds the permissible limit. ${ }^{1}$

As is obvious from the results, the power loss caused by turbulence reduces the link availability only in the case of relatively long link distances. Thus, the FSO designed for a relatively high availability in a typical continental area in Europe cannot be affected by turbulence. As mentioned above, the scintillation effect is reduced significantly by increasing the receiver aperture. A similar improvement can be obtained using a few optical transmitters. ${ }^{19}$

\section{Acknowledgments}

This work has been supported by the Research Programme MSM0021630513, and by the Grant Agency of the Czech Republic Projects No. 102/06/1358, No. 102/07/1295, and No. 102/09/0550.

\section{References}

1. I. I. Kim and E. Korevaar, "Availability of free space optics (FSO) and hybrid FSO/RF systems," Proc. SPIE 4530, 84-95 (2001).

2. L. C. Andrews and R. L. Phillips, Laser Beam Propagation through Random Media, SPIE, Bellingham (1998).

3. J. Alda, "Laser and Gaussian beam propagation and transformation," Encyclopedia of Optical Engineering, Marcel Dekker, New York (2003).

4. B. E. Saleh and M. C. Teich, Fundamentals of Photonics, Wiley, Hoboken, NJ (1991).

5. C. F. Bohren and D. Huffman, Absorption and Scattering of Light by Small Particles, Wiley, Hoboken, NJ (1983).

6. Guide to Meteorological Instruments and Methods of Observation and Information Dissemination, CIMO-XIV/Doc. 6.3, World Meteorological Organization, Geneva (1996).

7. P. W. Kruse, L. D. McGlauchlin, and R. B. McQuistan, Elements of Infrared Technology: Generation, Transmission, and Detection, Wiley, Hoboken, NJ (1962).

8. I. Kim, B. Mcarthur, and E. Korevaar, "Comparison of laser beam propagation at 785 and $1550 \mathrm{~nm}$ in fog and haze for opt. wireless communications," Proc. SPIE 4214, 26-37 (2001).

9. B. Flecker, M. Gebhart, E. Leitgeb, M. S. Sheikh, and C. Chlestil, "Results of attenuation-measurements for Optical Wireless channels under dense fog conditions regarding different wavelengths," Proc. SPIE 6303, 63030P (2006).

10. M. Naboulsi, H. Sizun, and F. de Fornel, "Fog attenuation prediction for optical and infrared waves," Opt. Eng., 43(2), 319-329 (2004).

11. O. Bouchet T. Marquis, M. Chabane, M. Al Naboulsi, and H. Sizun, "FSO and quality of service software prediction," Proc. SPIE 5892, 589204 (2005)

12. Z. Kolka, O. Wilfert, and V. Biolkova, "Reliability of Digital FSO Links in Europe," Int. J. Electronics, Communications, and Computer Engineering, 1(4), 236-239 (2007).

13. N. Perlot, "Evaluation of the scintillation loss for optical communication systems with direct detection," Opt. Eng. 46(2), 025003 (2007).

14. S. Rasouli and M. Tavassoly, "Measurement of the refractive-index structure constant, $C_{n}^{2}$, and its profile in the ground level atmosphere by moire technique," Proc. SPIE 6364, 63640G (2006).

15. G. d'Auria, F. S. Marzano, and U. Merlo, "Statistical estimation of mean refractive-index structure constant in clear air" in Proc. of 7th Int. Conf. Antennas and Propagation, 15-18 April 1991, York, UK, vol. 1, pp. 177-180, IEEE, Piscataway, NJ (1991).

16. G. R. Ochs, "Measurement of refractive-index structure parameter by incoherent aperture scintillation techniques," Proc. SPIE 1115, 107115 (1989)

17. L. C. Andrews, R. L. Phillips, and C. Y. Hopen, Laser Beam Scintillation with Applications, SPIE, Bellingham, WA, (2001).

18. V. I. Tatarski, The Effect of the Turbulent Atmosphere on Wave Propagation, Keter Press, Jerusalem (1961).

19. I. I. Kim, M. Mary, and E. J. Korevaar, "Measurement of scintillation for free-space laser communication at $785 \mathrm{~nm}$ and $1550 \mathrm{~nm}$," Proc. SPIE 3850, 49-62 (1999).

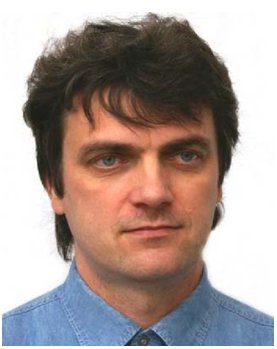

Ales Prokes received his $\mathrm{MSc}$ and $\mathrm{PhD}$ from Brno University of Technology, Czech Republic in 1988 and 2000, respectively. He is currently working as associate professor at the Institute of Radio Electronics of Brno University of Technology. His research interest includes nonuniform sampling and signal reconstruction, velocity measurement based on spatial filtering, beam propagation in atmosphere, and free-space optical communications. 